\title{
Combination of Color and Local Patterns as a Feature Vector for CBIR
}

\author{
L.Koteswara Rao \\ Asst.Professor, Dept of ECE \\ Faculty of Science and Technology \\ IFHE University \\ Hyderabad, India
}

\author{
D.Venkata Rao, Ph.D \\ Principal \\ Narasarao Pet Institute of Technology \\ Guntur District, Andhra Pradesh \\ India
}

\begin{abstract}
The local properties of an image can be acquired in many ways. Local Binary Patterns(LBP) operator is one among them in which a centre pixel is referenced with the neighboring pixels to obtain a feature vector. However, the directions are not considered in this method. The Directional Local Extrema Patterns(DLEP) are used to encode the relationship between the reference pixel and its neighbors by computing the edge information in four directions. In this paper, we propose a new approach based on the combination of DLEP and color to derive the properties those can be used in the process of retrieval.
\end{abstract}

\section{INTRODUCTION}

In recent years, due to the advances in the Internet and related fields, large number of images are being produced and stored across the globe. Hence there is a need for a system which can search and index these images in various applications. The conventional text based annotation method of image retrieval becomes inefficient when the database size is too large. Content based image retrieval(CBIR), has drawn the attention of researchers as an alternative to the existing methods in which, the visual contents such as color, texture, shape etc., are extracted for creation of feature vector .Similarity between set of features of query image and data base image is measured based on which more similar images are retrieved. However, the efficiency of any CBIR system depends on the extraction of features such as color, texture, shape etc., to index and retrieve the images [1][2].

It can be observed from the earlier works in the field of image retrieval that a single feature may not be sufficient to frame a feature vector. ,but use of many features may lead to more complexity in the process[3]. Among these features, color is one of the most discriminating and powerful descriptor which simplifies the object recognition [4] [5].Texture is another visual features that refers to the innate surface properties of an object and their relationship to the surrounding environment. Many approaches to classification and segmentation of texture based on statistical analysis, signal processing techniques were proposed in the past .In [6], use of texture feature for classification of images was discussed. Arivazhagan et al[7] proposed texture classification using wavelet transform. In [8] texture classification and segmentation was proposed using wavelet packet frames and Gaussian mixture model. Gabor wavelets were used in texture classification for rotation invariant features[9].

Discrete Wavelet Transform (DWT) with Kullback-Leibler distance was proved better alternative in getting more texture information [10]. However, a limitation of DWT based texture analysis is it can explore the features in $0^{\circ}, 45^{\circ}$ and $90^{\circ}$ directions only.

\subsection{Contribution}

The directional local extrema pattern (DLEP) extracts the directional edge information based on local extrema in $0^{\circ}$, $45^{\circ}, 90^{\circ}$, and $135^{\circ}$ directions in an image. The results can be further improved by combining this with color information. In this paper, we propose Combination of two features such as Texture and color to improve the performance of the existing Directional Local Extrema Pattern. The organization of this paper is as follows. Section 2 reviews about local patterns and variations. Section 3 explains the proposed work for retrieval system. Experimental Results are shown in Section 4. Section 5 represents the conclusion and future scope.

\subsection{Related work}

A feature extraction approach based on Local Binary Pattern was introduced by Ojala et. al [11].,Concept of LBP was extended to face recognition[12].However, LBP has the drawback of rotational invariance in classifying the structures. Local Derivative pattern by considering the $\mathrm{n}^{\text {th }}$ order Local Binary Pattern was proposed by Zhang et. al[13] Subramanyam et al[14] proposed Directional Local Extrema Pattern as a feature vector for texture analysis of an image. DLEP differs from the existing LBP and other extensions in terms of directional information.

\section{LOCAL PATTERNS AND VARIATIONS}

\subsection{Local Binary Pattern (LBP)}

Local binary pattern was introduced by $\mathrm{T}$ Ojala[11] .In this, the value of the centre pixel is considered as threshold. The difference between centre pixel value and its neighbor is taken into account to assign a binary 0 or 1 . The same procedure is repeated till all the neighbors surrounding the centre pixel are covered in the computation of the binary pattern.

$$
\mathrm{LBP}_{\mathrm{P}, \mathrm{R}}=\sum_{\mathrm{p}=0}^{\mathrm{p}-1} \mathrm{k}\left(\mathrm{g}_{\mathrm{p}}-\mathrm{g}_{\mathrm{c}}\right) 2^{\mathrm{p}}, \mathrm{k}(\mathrm{x})=\left\{\begin{array}{l}
1 \mathrm{x} \geq 0 \\
0 \mathrm{x}<0
\end{array}\right.
$$

where $g_{c}$ represents the gray value of the center pixel and $g_{p}$ corresponds to gray value of $\mathrm{P}$ equally spaced pixels on circumference of the circle with radius $\mathrm{R}$.

\subsection{Local Directional Pattern (LDP)}

Local Directional Pattern[15] is a method based on LBP which uses the edge response values of neighborhood pixels to encode the texture in an image. It assigns an eight bit binary code to each pixel of an input image.

A binary value of 1 or 0 is assigned depending on the presence of an edge. 
$\operatorname{LDP}_{\mathrm{n}}=\sum_{\mathrm{i}=1}^{8} \mathrm{f}_{\mathrm{i}}\left(\mathrm{m}_{\mathrm{i}}-\mathrm{m}_{\mathrm{k}}\right) * 2^{\mathrm{i}}, \mathrm{f}_{\mathrm{i}}(\mathrm{x})=\left\{\begin{array}{l}1, \mathrm{x} \geq 0 \\ 0, \mathrm{x}<0\end{array}\right.$

\subsection{Directional Local Extrema patterns (DLEP)}

DLEP was introduced by Subrahmanyam et al [14].It describes the spatial structure of the local texture using the local extrema of center gray pixel gc. The local extrema in four directions are obtained by calculating the difference between the centre pixel and all its neighbors.

In proposed DLEP, the local extrema in 00,450, 900 and 1350 directions are computed by taking the local difference between the center pixel and its neighbors as shown below.

$$
\mathrm{I}^{\prime}\left(\mathrm{g}_{\mathrm{i}}\right)=\mathrm{I}\left(\mathrm{g}_{\mathrm{c}}\right)-\mathrm{I}\left(\mathrm{g}_{\mathrm{i}}\right) ; \mathrm{i}=1,2, \ldots .8
$$

The local extremas are obtained using the equations given below.

$$
\begin{array}{r}
\hat{I}_{\alpha}(g c)=f_{3}\left(I^{\prime}\left(g_{i}\right) * I^{\prime}\left(g_{j+4}\right)\right) ; j=(1+\alpha / 45) \\
\forall_{\alpha}=0^{0}, 45^{0}, 90^{0}, 135^{\circ} \\
f_{3}\left(I^{\prime}\left(g_{j}\right), I^{\prime}\left(g_{j+4}\right)\right)= \begin{cases}1 & I^{\prime}\left(g_{j}\right) * I^{\prime}\left(g_{j+4}\right) \geq 0 \\
0 & \text { else }\end{cases}
\end{array}
$$

The DLEP is defined $\left(\alpha=0^{0}, 45^{0}, 90^{\circ}\right.$ and $\left.135^{\circ}\right)$ as follows:

$$
\left.\operatorname{DLEP}\left(\mathrm{I}\left(\mathrm{g}_{\mathrm{c}}\right)\right)\right|_{\alpha}=\left\{\hat{\mathrm{I}}_{\alpha}\left(\mathrm{g}_{\mathrm{c}}\right) ; \hat{\mathrm{I}}_{\alpha}\left(\mathrm{g}_{\mathrm{i}}\right) ; \hat{\mathrm{I}}_{\alpha}\left(\mathrm{g}_{\mathrm{z}}\right) ; \ldots . . . \mathrm{I}_{\alpha}\left(\mathrm{g}_{8}\right)\right)
$$

The detailed representation of DLEP can be seen in figure1.In the next step, the given image is converted to DLEP images with values ranging from 0 to 511 .

After calculation of DLEP, the whole image is represented by building a histogram based on the equation mentioned below.

$$
\begin{array}{r}
\mathrm{H}_{\text {DLEP } \mid \alpha}(\mathrm{i})=\sum_{\mathrm{j}=1}^{\mathrm{N}_{1}} \sum_{\mathrm{k}=1}^{\mathrm{N}_{2}} \mathrm{f}_{2}\left(\left.\operatorname{DLEP}(\mathrm{j}, \mathrm{k})\right|_{\alpha}, \ell\right) ; \\
\ell \in[0,511]
\end{array}
$$

where the size of input image is $\mathrm{N}_{1} \times \mathrm{N}_{2}$. The procedure for calculation of DLEP for center pixel marked with green color is presented in fig.1.The directions are evaluated using the local difference between the center pixel and its neighbors from which the DLEPs are obtained.

As an example, the DLEP in 900 direction for a center pixel marked in green color is shown in the figure2. For a center pixel value 27 , it can be observed that two neighboring pixels are leaving. Therefore, this pattern is represented as 1 . In the same way the rest of the bits of DLEP pattern are computed and the outcome is 110011110. Similarly, the DLEP's are computed for $0^{\circ}, 45^{\circ}$ and $135^{\circ}$ directions.

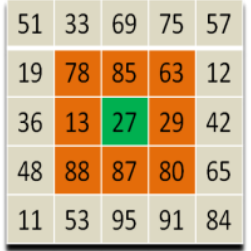

\begin{tabular}{|l|l|l|l|l|l|l|l|l|l|l|}
\hline & $0_{(27)}$ & $1_{(29)}$ & $2_{(80)}$ & $3_{(87)}$ & $4_{(88)}$ & $5_{(13)}$ & $6_{(78)}$ & $7_{(85)}$ & $8_{(63)}$ & DLEP \\
\hline $\mathrm{P}\left(0^{0}\right)$ & 0 & 0 & 0 & 0 & 1 & 1 & 0 & 1 & 0 & 26 \\
\hline $\mathrm{Q}\left(45^{0}\right)$ & 1 & 0 & 0 & 1 & 1 & 1 & 1 & 0 & 1 & 317 \\
\hline $\mathrm{R}\left(90^{0}\right)$ & 1 & 1 & 0 & 0 & 1 & 1 & 1 & 1 & 0 & 415 \\
\hline $\mathrm{S}\left(135^{\circ}\right)$ & 1 & 1 & 0 & 0 & 0 & 1 & 1 & 1 & 0 & 398 \\
\hline
\end{tabular}

Fig. 1 Illustration of DLEP for $3 \times 3$ pattern
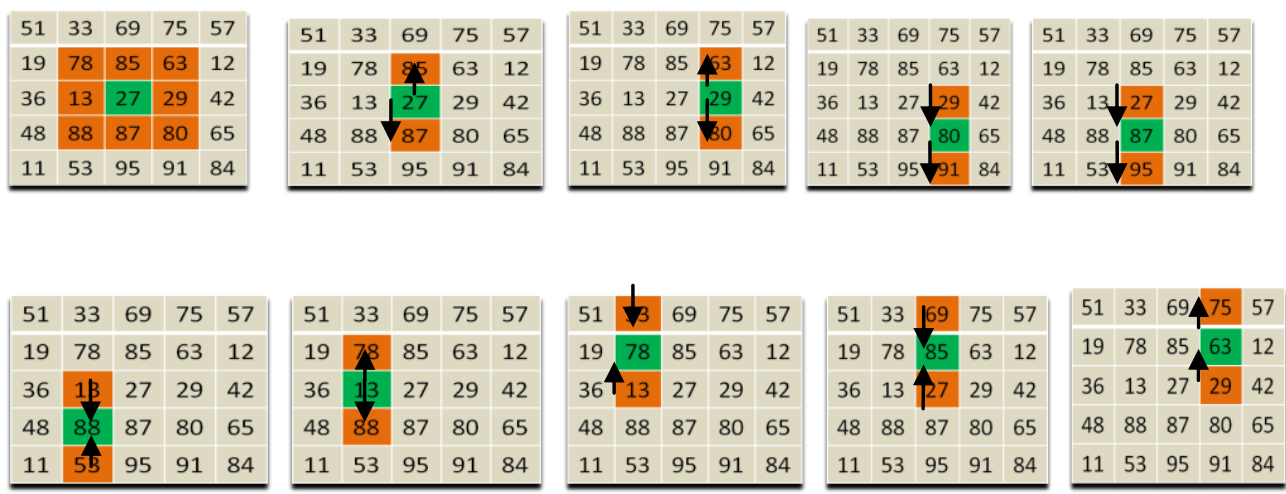

Fig. 2 Example to compute DLEP in 900 direction (110011110) 


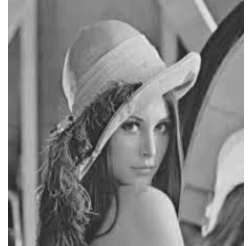

(a)

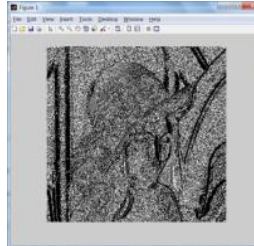

(b)

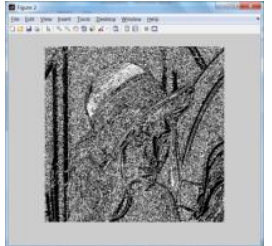

(c)

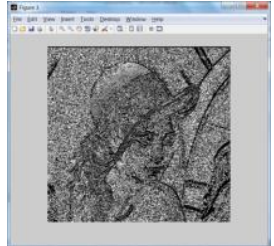

(d)

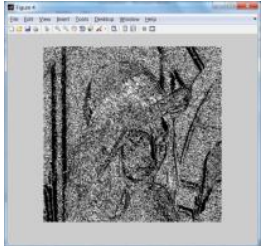

(e)

Figure3: Example of DLEP maps; (a) Sample Image (b) 00 (c) 450 (d) 900 (e) 1350

\section{PROPOSED CDLEP SYSTEM}

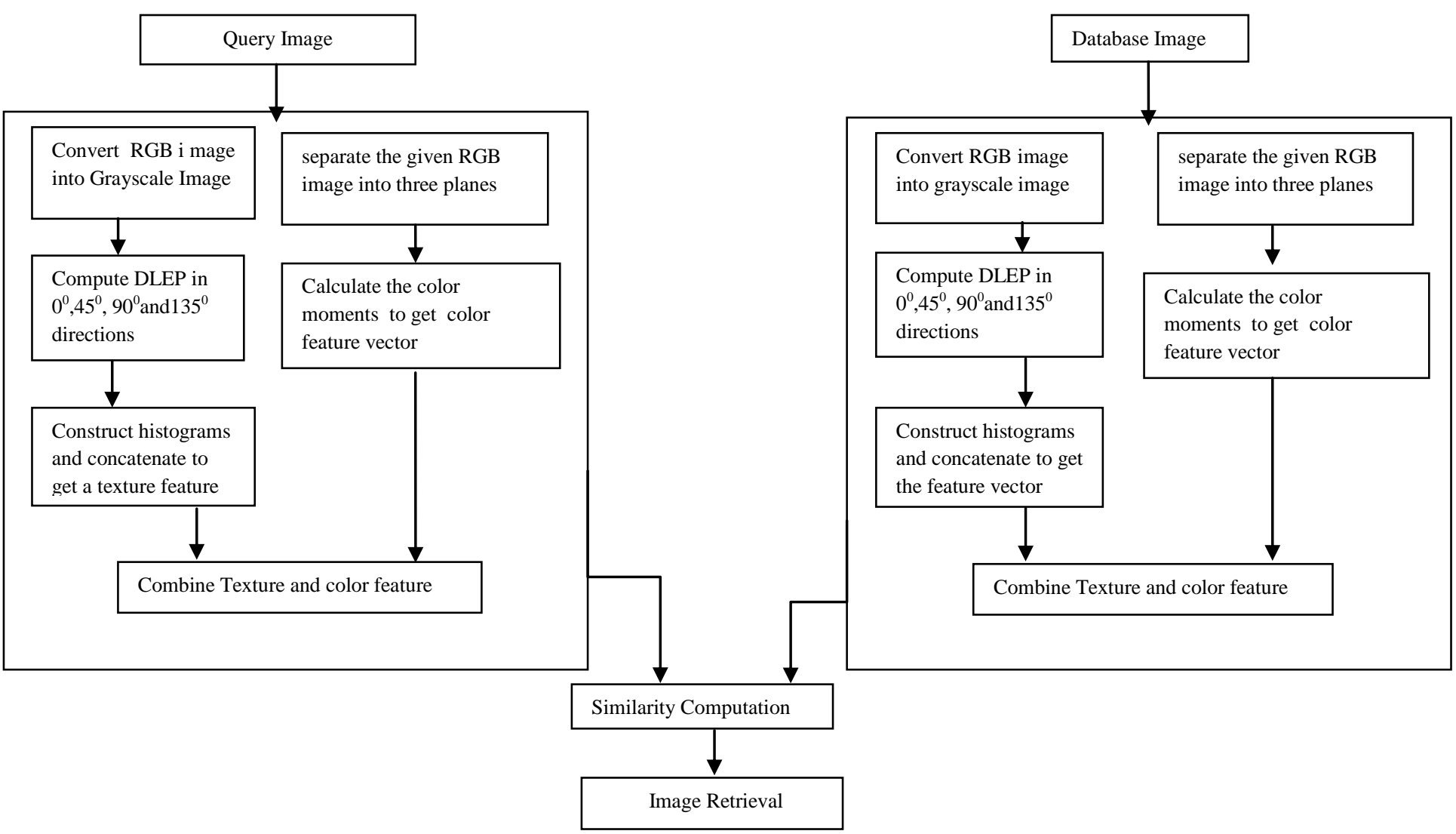

Fig.4 Proposed framework for content based Image retrieval

\subsection{Algorithm}

i. Convert the RGB image into gray scale image.

ii. Compute the local extrema in $0^{0}, 45^{\circ}, 90^{\circ}$ and $135^{\circ}$ directions.

iii. Calculate the DLEP patterns in four directions mentioned in step 2 .

iv. Make a histogram for the DLEP patterns obtained in step 3 and concatenate to get the texture feature vector

v. To get the feature vector based on color, take the color moments in R,G,B planes separately and make a feature vector

vi. Combine these two features to get a feature vector that can be used in image retrieval.

\subsection{Query Matching}

After the extraction of features, the feature vector for query image is computed. In the same way, feature vector for the images in the database is also computed. In order to select the more relevant image to the query image, the distance between query and database images is computed.

\section{EXPERIMENTAL RESULTS}

Performance of the CDLEP is evaluated on standard corel- $1 \mathrm{k}$ database [16]. The precision $(\mathrm{P})$ and recall $(\mathrm{R})$ values are computed as per the relationship mentioned here under.

$$
\begin{gathered}
P=\frac{\text { No. of relevant images retrieved }}{\text { No. of images retrieved }} \\
R=\frac{\text { No. of relevant images retrieved }}{\text { No. of relevant images in the database }}
\end{gathered}
$$


The results for ten categories of the data base are specified in the table 1 .

Table 1: Comparison of precision values for DLEP and CDLEP

\begin{tabular}{|l|l|l|}
\hline Category & $\begin{array}{l}\text { Existin } \\
\text { g } \\
\text { DLEP }\end{array}$ & $\begin{array}{l}\text { DLEP+ } \\
\text { Color } \\
\text { feature }\end{array}$ \\
\hline Africans & 69.3 & 80 \\
\hline Beach & 60.5 & 80 \\
\hline Building & 72.0 & 100 \\
\hline Buses & 97.9 & 90 \\
\hline Dinosaur & 98.5 & 100 \\
\hline Elephant & 55.9 & 80 \\
\hline Flower & 91.9 & 100 \\
\hline Horse & 76.9 & 100 \\
\hline Mountain & 42.7 & 60 \\
\hline Food & 82.0 & 90 \\
\hline $\begin{array}{l}\text { Average } \\
\text { Precision } \\
\text { (\%) }\end{array}$ & $\mathbf{7 4 . 8}$ & $\mathbf{8 8 . 0}$ \\
\hline
\end{tabular}

Table 2: Comparison of recall values for DLEP and CDLEP

\begin{tabular}{|l|l|l|}
\hline Category & DLEP & $\begin{array}{l}\text { DLEP+ } \\
\text { Color } \\
\text { feature }\end{array}$ \\
\hline Africans & 39.7 & 41 \\
\hline Beach & 37.3 & 39 \\
\hline Building & 34.9 & 41 \\
\hline Buses & 74.1 & 67 \\
\hline Dinosaur & 88.0 & 85 \\
\hline Elephant & 29.0 & 34 \\
\hline Flower & 70.8 & 83 \\
\hline Horse & 41.7 & 42 \\
\hline Mountain & 29.0 & 30 \\
\hline Food & 47.0 & 43 \\
\hline $\begin{array}{l}\text { Average } \\
\text { Recall (\%) }\end{array}$ & 49.16 & 50.5 \\
\hline
\end{tabular}

The comparisons in terms of average precision and recall are given in the graph given below.

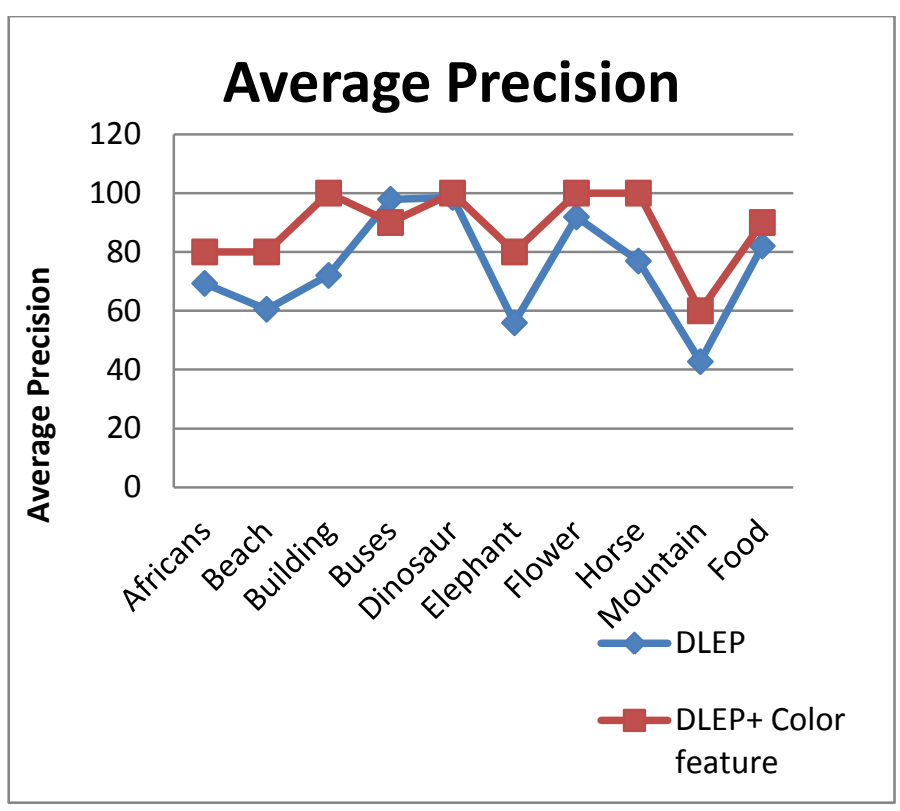

Figure 5. category- wise performance in terms of precision

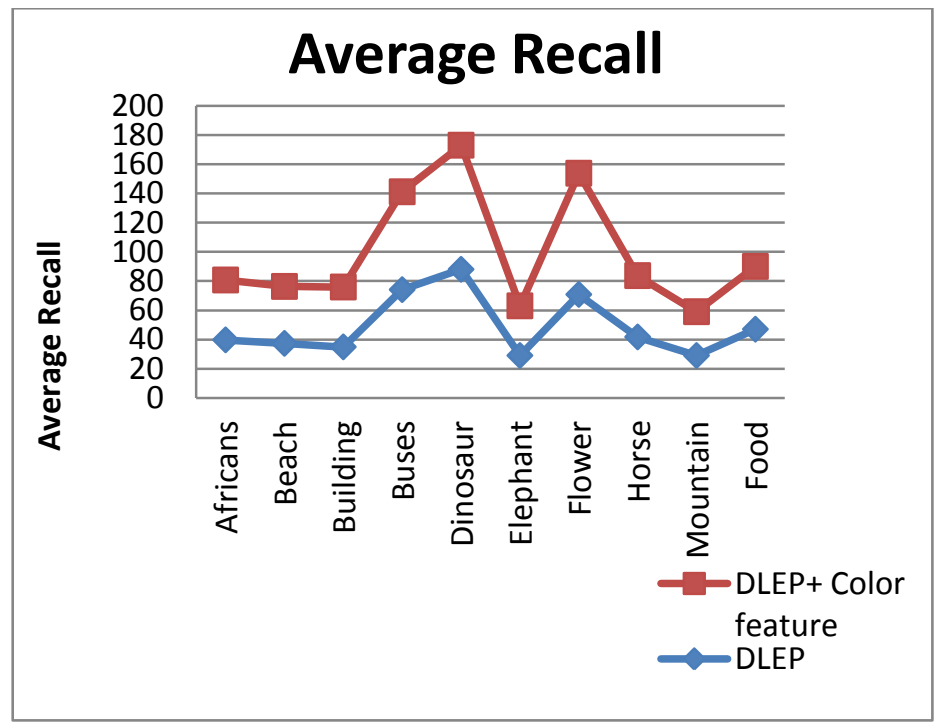

Figure 6. category- wise performance in terms of recall

\section{CONCLUSION AND FUTURE SCOPE}

It is proved from the results that the precision and Recall values of the proposed method are better than the existing directional patterns. This work can be further extended by combining the CDLEP with transforms such as Contourlets or Curvelet transforms to get the texture information in more directions.

6. REFERENCES

[1] R. Datta, D Joshi J.Li and J. Wang,' Image RetrievalIdeas, influences and trends of the new age', ACM Computing surveys,vol.40,no.2,pp 1-60 2008

[2] AM smeulders,M Worring,S Santini,A Gupta\&R Jain,"content based Image retrieval at the end of early years"IEEE Transactions aon PAMI 22(12),pp 13491380,2000 
[3] Y Rui,T S Huang\&S F Chang," Image retrieval: current techniques, promising directions\& open issues. Journal of visual communications\& Image representation 10(4):pp 39-62,1999.

[4] Digital Image Processing Gonzalez\& Woods third edition

[5] M J Swain,D H Ballard, Color Indexing, International Journal of computer vision (1991) 11-32

[6] R M Haralick,K Shanmugam\&i dinstein," texture features for image classification ",IEEE transactions on system, man and cybernetics vol,smc-8,pp 610-621, 1973

[7] S Arivazhagan and L Ganesan, "Texture classification using wavelet transform(1513-1521) vol.24,issue 910,June 2003

[8] Soo chang Kim,Tae Jin Kang,"Texture classification and segmentation using wavelet packet frame and Gaussian mixture model vol 40,issue 4,april 2007, 1207-1221 elsevier

[9] S Arivazhagan and L Ganesan, 'Texture classification using Gabor wavelets based rotation invariant features ,vol 27,ISSUE 16, December 2006 (1976-82)
[10] Do MN,Vettrli M(2002) Wavelet-based texture retrieval using generalized Gaussian density and Kullback-leibler distance, IEEE Transactions on Image processing.vol 11(2).

[11] Ojala T,Pietikainen M,Harwood D (1996) A comparative study of texture measures with classification based on feature distributions. J Pattern Recognition 29(1):51-59

[12] A Hadid,T Ahonen and M Pietikainen,"Face analysis using local binary patterns," in handbook of Texture analysis, Imperial college press London 2008,pp 347-373

[13] Zhang B, Gao Y,Zhao S, Liu J (2010) Local derivative pattern versus local binary pattern: Face Recognition with higher-order local pattern descriptor, IEEE Trans Image Process 19(2):533-544

[14] Subrahmanyam Murala, R.P. Maheswari, R.Balasubramanian Directional local extrema pattern: a new descriptor for content based image retrieval(2012)

[15] Jabid,,Kabir, Chae Kyung H, "Local Directional Pattern for face recognition" IEEE conference 2010 